\title{
Strange and identified particle production with ALICE at the LHC
}

\author{
Domenico Elia ${ }^{1, a}$, on behalf of the ALICE Collaboration \\ ${ }^{1}$ INFN Sezione di Bari, via Orabona 4, 70126 Bari, Italy
}

\begin{abstract}
The ALICE experiment has measured the production of strange and identified charged particles in $\mathrm{pp}, \mathrm{p}-\mathrm{Pb}$ and $\mathrm{Pb}-\mathrm{Pb}$ collisions at LHC energies. Light-flavour hadrons are identified in various momentum ranges by using specific energy loss, timeof-flight, Cherenkov radiation, as well as decay topology and invariant mass analysis for weakly-decaying strange particles.

In this contribution, the transverse momentum spectra at mid-rapidity and the corresponding yields measured in $\mathrm{pp}, \mathrm{p}-\mathrm{Pb}$ and $\mathrm{Pb}-\mathrm{Pb}$ collisions for different multiplicity or centrality intervals are presented. The production of light-flavour hadrons in the three collision systems is compared and confronted with theoretical models.
\end{abstract}

\section{Introduction}

Heavy-ion collisions at ultra-relativistic energies allow one to study the physics of the strongly interacting matter and to characterize the Quark-Gluon Plasma (QGP) [1]. The hot and dense matter created in such collisions expands due to the pressure gradients between the system centre and the surrounding vacuum: the system expands collectively and cools down and then undergoes hadronization. Transverse momentum spectra of identified hadrons at low $p_{\mathrm{T}}$ (below $3 \mathrm{GeV} / c$ ) provide information about the collective behaviour of the system (e.g. effects of the radial flow). The intermediate transverse momentum region $\left(3<p_{\mathrm{T}}<8 \mathrm{GeV} / c\right)$ allows one to investigate hadronization mechanisms according to models like quark-recombination [2], while at higher $p_{\mathrm{T}}$ (above $8 \mathrm{GeV} / c$ ) mediuminduced effects on the fragmentation can be investigated [3]. Furthermore, the production of strange particles in ultra-relativistic heavy-ion interactions provides a unique additional tool to investigate the properties of the system created in the collision, as there is no net strangeness content in the initially colliding nuclei. In particular, an enhanced production of strange particles in A-A compared to pp interactions was one of the earliest proposed signatures of a deconfined QGP phase $[4,5]$.

Measurements in pp collisions performed by ALICE serve not only as baseline for heavy-ion reactions but also as a valuable handle on the particle production mechanisms at play and a tool for the tuning of Monte Carlo generators at LHC energies. Corresponding studies in $\mathrm{p}-\mathrm{Pb}$ collisions have been carried out as well, with the main aim of performing a control experiment to study initial cold nuclear matter effets. The proton-nucleus reactions have also been able to provide new interesting and partly unexpected physics results. In the next sections, following a brief description of the ALICE detector and the analysis techniques used to reconstruct strange and identified particles, selected results on light-flavour hadrons produced in the three collision systems for a wide range of transverse momentum are presented and the corresponding physics implications are discussed.

\footnotetext{
a e-mail: Domenico.Elia@ba.infn.it
} 


\section{ALICE detector and light-flavour hadron measurement}

The ALICE experiment is designed to study the properties and the evolution of the hot and dense matter created in heavy-ion collisions at the LHC. The apparatus, schematically shown in Fig. 1, consists of a central barrel with a large solenoid providing a $0.5 \mathrm{~T}$ magnetic field for tracking and particle identification, plus a dimuon spectrometer equipped with its own dipole magnet with a 0.7 Tm field integral, and other forward detectors for triggering and centrality selection. Tracking and vertexing are performed using the Inner Tracking System (ITS), consisting of six layers of silicon detectors, and the Time Projection Chamber (TPC). The two innermost layers of the ITS and the VZERO detector (scintillation hodoscopes placed on either side of the interaction region) are used for triggering. The VZERO also provides the centrality (multiplicity) class definition in $\mathrm{Pb}-\mathrm{Pb}(\mathrm{p}-\mathrm{Pb})$ collisions. A complete description of the ALICE sub-detectors can be found in $[6,7]$.

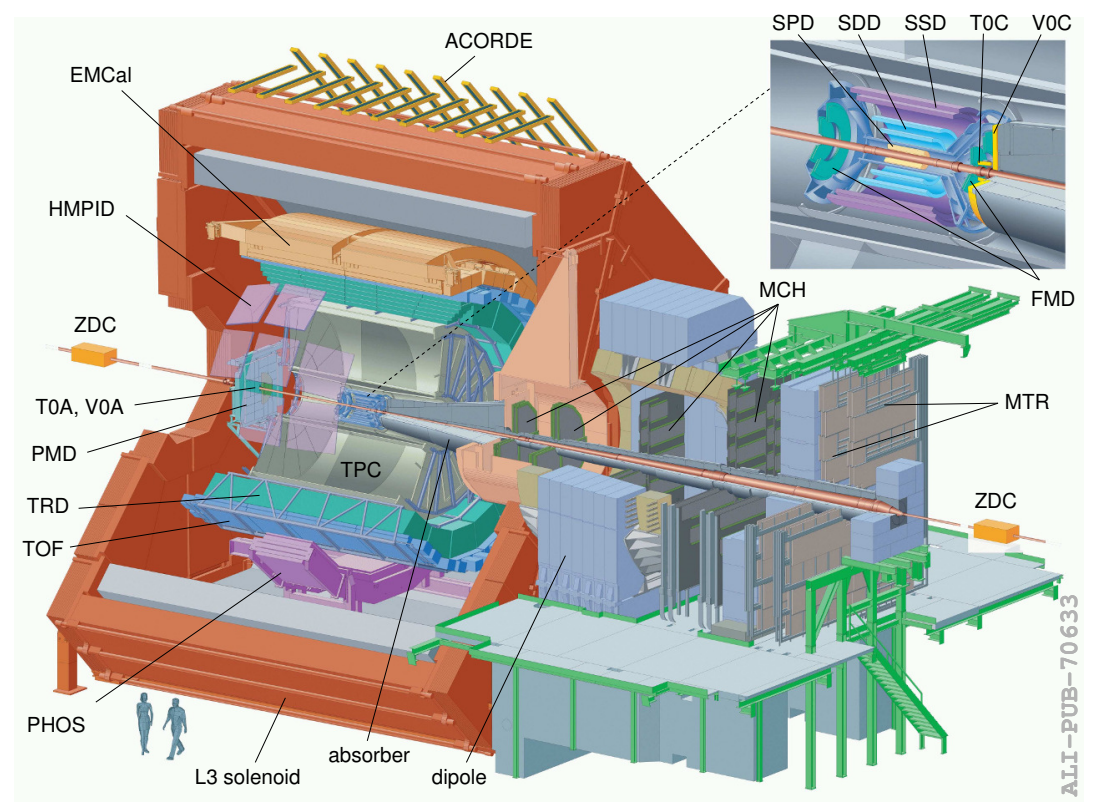

Figure 1. Schematic view of the ALICE experimental apparatus.

Unique among the LHC experiments, ALICE has excellent particle identification capabilities in a broad transverse momentum range from 0.1 to $20 \mathrm{GeV} / c$. Charged hadrons in the low to intermediate$p_{\mathrm{T}}$ region (below $5 \mathrm{GeV} / c$ ) are identified using the energy loss $\mathrm{d} E / \mathrm{d} x$ from the ITS and TPC detectors, the time-of-flight measurement from the TOF detector and the Cherenkov light from the High Momentum Particle Identification Detector (HMPID). At higher $p_{\mathrm{T}}$ charged particles are identified via the $\mathrm{d} E / \mathrm{d} x$ in the relativistic rise range of the Bethe-Bloch curve in the TPC. Strange hadrons are measured through the reconstruction of their weak decays into charged particles. $\mathrm{K}_{S}^{0}, \Lambda$ and $\bar{\Lambda}$ candidates are found by combining two charged tracks into $\mathrm{V}$-shaped decays ("V ${ }^{0}$ " topology), while for multi-strange baryons (charged $\Xi$ and $\Omega$ ) a selected $\mathrm{V}^{0}(\Lambda$ candidate) is further combined with a third track. Candidates are required to satisfy topological and kinematic restrictions. In addition, each of the daughter tracks is checked for compatibility with the pion, kaon or proton hypotheses using their energy loss in the TPC. Further details, including the invariant mass analysis and signal extraction procedure for strange weakly-decaying particles, can be found in $[8,9]$. 


\section{Results and discussion}

Results presented in this contribution are based on the following data samples: pp collisions at $\sqrt{s}=7$ $\mathrm{TeV}$ collected in $2010, \mathrm{~Pb}-\mathrm{Pb}$ collisions at $\sqrt{s_{\mathrm{NN}}}=2.76 \mathrm{TeV}$ from the 2010 and 2011 runs and $\mathrm{p}-\mathrm{Pb}$ events at $\sqrt{s_{\mathrm{NN}}}=5.02 \mathrm{TeV}$ recorded at the beginning of 2013. The main results on the measurement of strange particle production in ALICE for the three collision systems are described in the following sub-sections. A few selected aspects of the corresponding findings for identified charged particles are also discussed, while additional details can be found elsewhere in these proceedings [10].

\subsection{Results in pp collisions}

The transverse momentum spectra at low $p_{\mathrm{T}}$ for $\pi, \mathrm{K}$ and $\mathrm{p}$ in pp collisions at $\sqrt{s}=7 \mathrm{TeV}$ have been extracted by selecting events in eight multiplicity classes defined by the raw charged-particle multiplicity. The scaled multiplicity $z_{\text {raw }}$ (ratio of the average multiplicity in a given class to that for events with multiplicity larger than zero) has been used for partitioning the sample. The lowest and highest multiplicity bins correspond to $z_{\text {raw }}$ in the intervals $[0.7,1.4)$ and $[7.5,8.6]$, respectively. The resulting spectra are presented in the top row of Fig. 2, while in the bottom row of the same figure the ratios of the $p_{\mathrm{T}}$ distributions at different multiplicity relative to the mult $>0$ case are shown. A systematic change in the shape of the spectra, with a depletion of the low- $p_{\mathrm{T}}$ region and a tendency to level off at larger momentum values, is observed. The amount of depletion depends both on the particle mass and on the multiplicity, being enhanced going from pions to protons and with increasing multiplicity for a given particle.
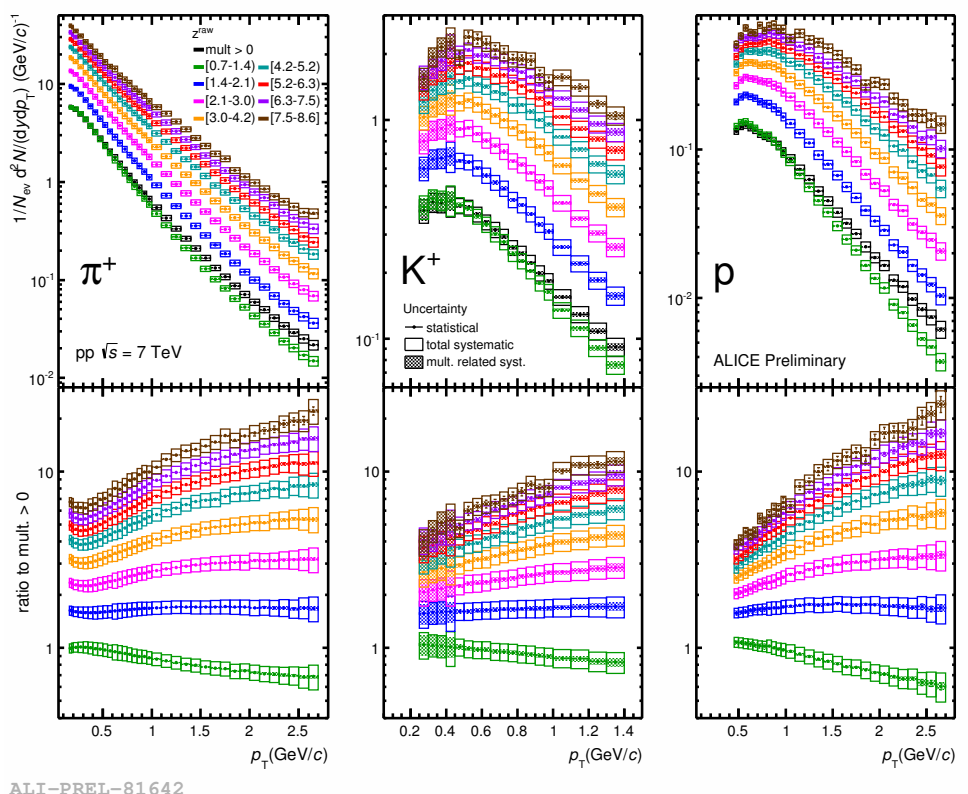

Figure 2. Top row: charged-particle multiplicity dependence of the transverse momentum distributions for $\pi^{+}$, $\mathrm{K}^{+}$and $\mathrm{p}$ in pp collisions at $\sqrt{s}=7 \mathrm{TeV}$. Bottom row: ratios of the tranverse momentum distributions in different multiplicity bins to the distribution for the mult $>0$ case. 
The production at mid-rapidity of $\mathrm{K}_{S}^{0}, \Lambda$ and $\bar{\Lambda}$ in pp collisions at $\sqrt{s}=7 \mathrm{TeV}$ has been also studied: the corresponding transverse momentum spectra are shown in Fig. 3. Particle and antiparticle distributions are compatible within the uncertainties over the measured $p_{\mathrm{T}}$ range. The $p_{\mathrm{T}^{-}}$ integrated production yields are obtained by fitting the spectra with the Tsallis function: the fraction in the unmeasured region is about $15 \%$ for $\Lambda$ and negligible for $\mathrm{K}_{S}^{0}$. The spectra have been compared with predictions from PYTHIA Perugia-2011 [11, 12], which provides the best description for the multi-strange baryons [9] and has been tuned to the measured charged-particle multiplicity at the same centre-of-mass energy. As can be seen in the bottom panel of Fig. 3, the agreement is rather good in the high- $p_{\mathrm{T}}$ region (above $6 \mathrm{GeV} / c$ ), while in the soft part PYTHIA underestimates the spectra by approximately $20 \%$ for $\mathrm{K}_{S}^{0}$ and more than a factor of two for $\Lambda$.

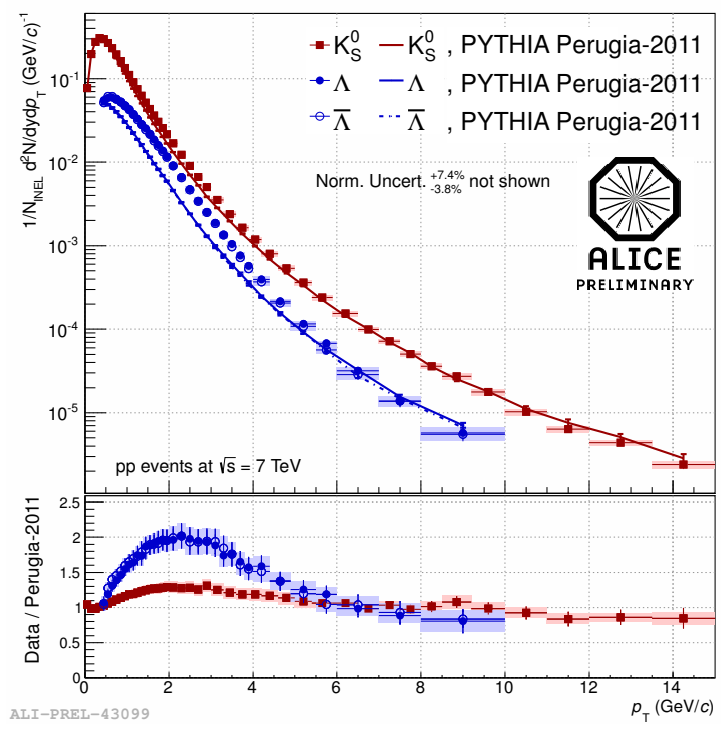

Figure 3. Top panel: transverse momentum spectra for $\Lambda, \bar{\Lambda}$ and $\mathrm{K}_{S}^{0}$ (symbols) in pp collisions at $\sqrt{s}=7 \mathrm{TeV}$, compared to PYTHIA Perugia-2011 predictions (lines). Bottom panel: ratios between data and PYTHIA Perugia2011 distributions.

A larger disagreement between the PYTHIA Perugia-2011 predictions and data can be seen in the top left panel (a) of Fig. 4 for the multi-strange baryon spectra. The bottom left panel (b) of Fig. 4 shows that, while being the best available tune, PYTHIA Perugia-2011 underestimates up to a factor of 2 and 5 the $\Xi$ and $\Omega$ spectra, respectively, in the intermediate transverse momentum region. At high $p_{\mathrm{T}}$ the discrepancy decreases for $\Xi^{-}$and $\bar{\Xi}^{+}$when entering the fragmentation regime, while no conclusion can be drawn for $\Omega^{-}$and $\bar{\Omega}^{+}$with the available statistics. Similar results have also been found for multi-strange baryons in pp collisions at $\sqrt{s_{\mathrm{NN}}}=2.76 \mathrm{TeV}$ [13]. In the right panel of Fig. 4, the multi-strange baryon yields and $\left\langle p_{\mathrm{T}}\right\rangle$ are shown for ALICE data at $\sqrt{s}=0.9 \mathrm{TeV}$ and $7 \mathrm{TeV}$ [8,9] and STAR at $\sqrt{s}=0.2 \mathrm{TeV}$ [14]. It can be observed that PYTHIA Perugia-2011 roughly reproduces the energy dependence of the multi-strange baryon production: this has been used to interpolate the pp yields to the reference energy in the calculation of the strangeness enhancements for $\mathrm{Pb}-\mathrm{Pb}$ collisions presented in the next section. 

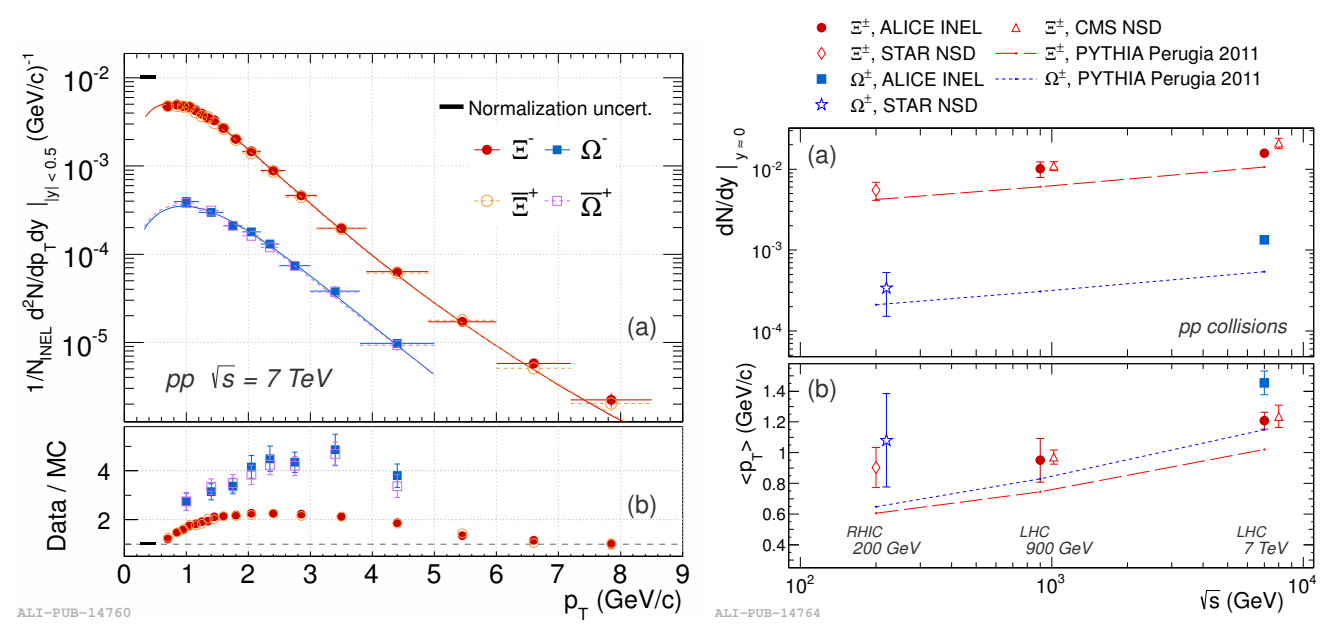

Figure 4. Left: transverse momentum spectra for $\Xi^{-}$and $\Omega^{-}$baryons (full symbols) and their anti-particles (open symbols) in pp collisions at $\sqrt{s}=7 \mathrm{TeV}$, compared to PYTHIA Perugia-2011 predictions (lines) [9]. Right: multi-strange baryon yields (a) and $\left\langle p_{\mathrm{T}}\right\rangle$ (b) as a function of centre-of-mass energy in pp collisions.

\subsection{Results in $\mathrm{Pb}-\mathrm{Pb}$ collisions}

A broad range of measurements has been performed in $\mathrm{Pb}-\mathrm{Pb}$ collisions at $\sqrt{s_{\mathrm{NN}}}=2.76 \mathrm{TeV}$. Strange and identified hadron spectra have been measured in different centrality intervals according to the fraction of the total inelastic cross-section. The definition of the event centrality is based on the sum of the amplitudes measured in the VZERO detectors, as described in [15].

The transverse momentum spectra for pions, kaons and (anti-)protons (sum of both charges) in the full $p_{\mathrm{T}}$ range up to $20 \mathrm{GeV} / c$ are shown in Fig. 5, both for central (0-5\%) and peripheral (60$80 \%) \mathrm{Pb}-\mathrm{Pb}$ collisions [16]. Compared with the pp reference scaled by the average number of binary collisions in each centrality class, the spectra measured in central $\mathrm{Pb}-\mathrm{Pb}$ collisions show a reduction of particle production at high $p_{\mathrm{T}}$ which can be qualitatively explained as an effect of jet quenching. Similar shapes as for $\mathrm{pp}$ are indeed observed for the $60-80 \%$ peripheral $\mathrm{Pb}-\mathrm{Pb}$ collision spectra.

The collective behaviour of the system created in ultra-relativistic heavy-ion collisions can be described by hydrodynamical models. Spectra of identified hadrons at low $p_{\mathrm{T}}$ are a major constraining factor for those models. Results for pions, kaons and protons measured in central $\mathrm{Pb}-\mathrm{Pb}$ collisions show a good agreement with model predictions and a higher mean $p_{\mathrm{T}}$ compared to RHIC [17]. This is considered as a signature of a stronger radial flow at LHC energies. Comparisons with predictions from hydrodynamic calculations, based on the same models used for $\pi, \mathrm{K}$ and p (VISH2+1 [18], HKM [19], Kraków [20] and EPOS [21]), have also been carried out for the heavier multi-strange baryons [22]. The results are shown in Fig. 6 for $\Xi$ and $\Omega$ hyperons (average of both charges) in different ranges of centrality. For the most central (0-10\%) events, all the available models describe quite well the shape of the $\Xi$ spectrum in the $p_{\mathrm{T}}$ range up to $3 \mathrm{GeV} / c$, although only the Kraków model correctly reproduces the yield. The description is less successful overall with the $\Omega$. Moving progressively to less central events, the quality of the agreement remains similar for the $\Xi$, but deteriorates for the $\Omega$. Comparing these models gives an insight into the mechanism at work in hyperon production. VISH2+1, which results in the least successful description, does not include the hadronic cascade mechanism. The EPOS generator, which incorporates hydrodynamics and models the inter- 


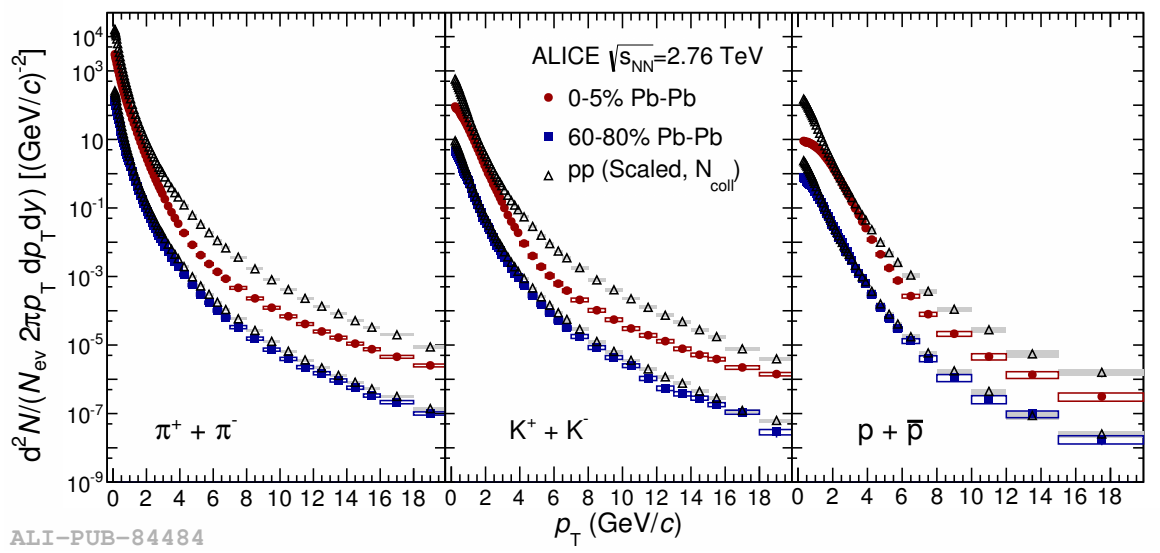

Figure 5. Transverse momentum spectra for charged identified particles in 0-5\% (full circles) and $60-80 \%$ (full squares) centrality $\mathrm{Pb}-\mathrm{Pb}$ collisions at $\sqrt{s_{\mathrm{NN}}}=2.76 \mathrm{TeV}$ [16]. Open triangles represent the corresponding pp reference yields scaled by the average numbers of binary collisions in the two centrality classes. Statistical and systematic uncertainties are reported as vertical error bars and boxes, respectively.

action between high- $p_{\mathrm{T}}$ hadrons and the expanding fluid, gives the most successful description overall in a wider transverse momentum range.
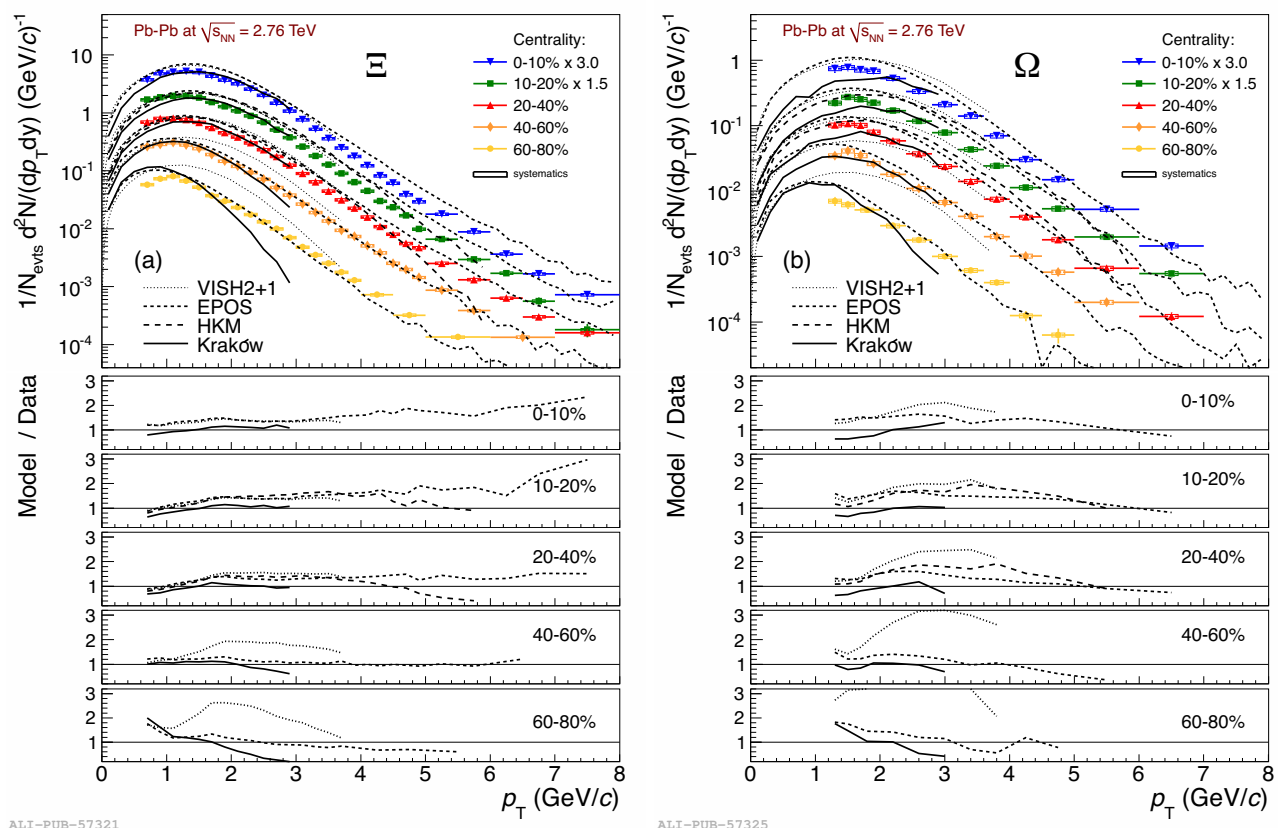

Figure 6. Transverse momentum spectra for $\Xi$ (a) and $\Omega$ (b) hyperons (average of particle and anti-particle) for $\mathrm{Pb}-\mathrm{Pb}$ collisions at $\sqrt{s_{\mathrm{NN}}}=2.76 \mathrm{TeV}$ in five centrality classes, compared to hydrodynamical models [22]. 
In high-energy interactions hadrons are expected to be produced in approximate chemical and thermal equilibrium. A grand-canonical statistical model is usually employed to describe the observed yields of each particle specie in nucleus-nucleus collisions. The mid-rapidity $p_{\mathrm{T}}$-integrated yields measured by ALICE in central $\mathrm{Pb}-\mathrm{Pb}$ collisions at $\sqrt{s_{\mathrm{NN}}}=2.76 \mathrm{TeV}$ have been fitted to three statistical hadronization models, namely THERMUS 2.3 [23], GSI-Heidelberg [24] and SHARE [25]. Chemical freeze-out temperature and fireball volume have been fitted to the data, with the baryochemical potential being set to $1 \mathrm{MeV}$ since particle and anti-particle yields are equal within the uncertainties: the results are illustrated in Fig. 7.

All three models provide the same temperature, $T_{\text {chem }} \approx 155-156 \mathrm{MeV}$, and reproduce most of the measured yields. Nevertheless, they overestimate the proton and anti-proton yields. The interpretation of this tension, one of the most discussed feature of heavy-ion collision results at the LHC, is still under investigation and could be explained in three different approaches: baryon-antibaryon annihilation after chemical freeze-out [26], non-equilibrium statistical hadronization [27] and effects due to pre-hadronic flavour-dependent bound states above the QCD transition temperature [28]. The $\mathrm{K}^{*}$ resonance yield is also overestimated: excluding both protons and $\mathrm{K}^{*}$ from the fit restores the consistency with equilibrium expectations [29].

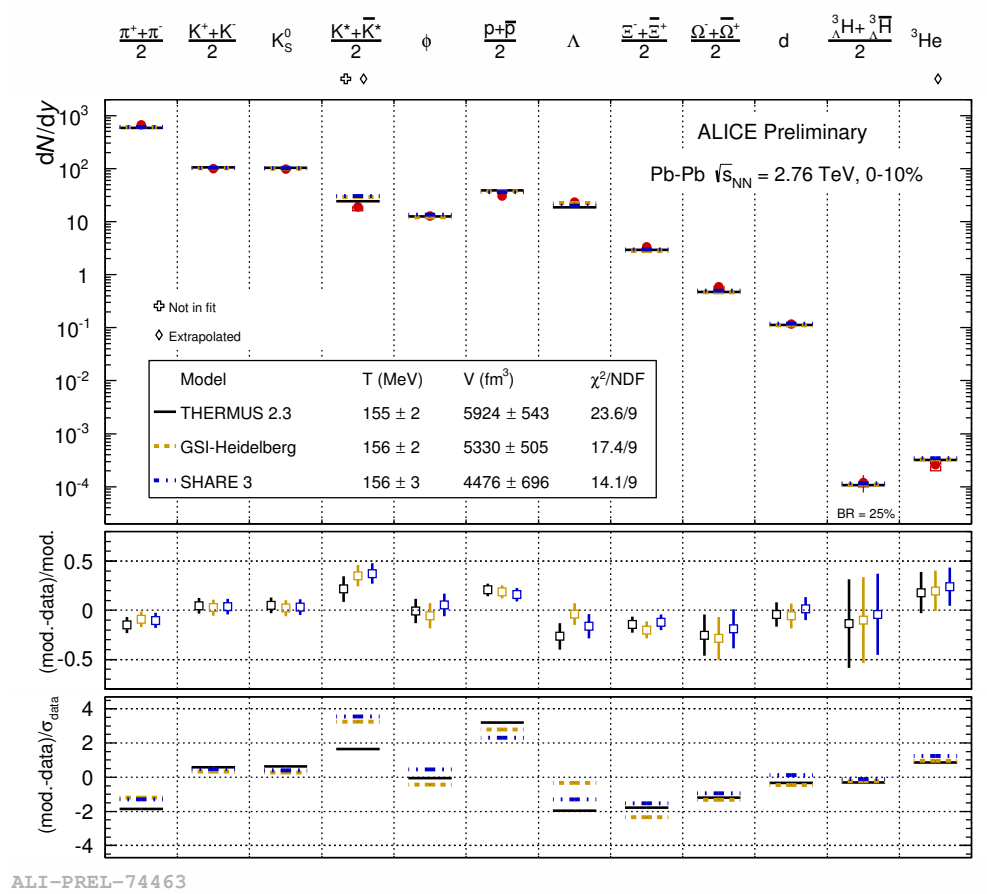

Figure 7. Thermal fits of mid-rapidity particle yields in $0-10 \%$ central $\mathrm{Pb}-\mathrm{Pb}$ collisions at $\sqrt{s_{\mathrm{NN}}}=2.76 \mathrm{TeV}$ to three different implementations of statistical hadronization model.

Among the most interesting results involving strange particles, the strangeness enhancements at the LHC have been also measured. As already mentioned, the enhancement of strange and multistrange particle production in heavy-ion collisions compared to pp was one of the earliest proposed signatures for the QGP $[4,5]$. It was argued that a deconfined partonic phase in the system evolution implies a considerable increase in strange quark phase space occupancy compared to the observed 
strangeness production levels in pp interactions. Indeed, an enhanced production of strange particles has been observed both at SPS [30, 31] and RHIC [32] energies. The strangeness enhancements are defined as ratios of the strange particle yields measured in $\mathrm{Pb}-\mathrm{Pb}$ collisions, normalized to the mean number of participant nucleons $\left\langle N_{\text {part }}\right\rangle$, to the corresponding quantities in pp interactions at the same energy. The ALICE results for $\Lambda(|S|=1), \Xi^{-}(|S|=2)$ and $\Omega^{-}+\bar{\Omega}^{+}(|S|=3)$ are shown in the left panel of Fig. 8 as a function of $\left\langle N_{\text {part }}\right\rangle$. The pp reference values were obtained by interpolating ALICE and STAR data [22]. The enhancement factors are larger than unity for all the particles and increase with $\left\langle N_{\text {part }}\right\rangle$. They also increase with the strangeness content of the particle, showing the hierarchy already observed at lower energies and consistent with the picture of enhanced $s \bar{s}$ pair production in a hot and dense partonic medium. In the right panel of Fig. 8 the ALICE results are compared with lower energy measurements performed at SPS and RHIC: the enhancements are seen to decrease with increasing centre-of-mass energy, continuing the same trend first established at the SPS and then confirmed going from SPS to RHIC energies. Such an energy dependence pattern is mostly attributed to the decrease of canonical suppression in the pp reference.
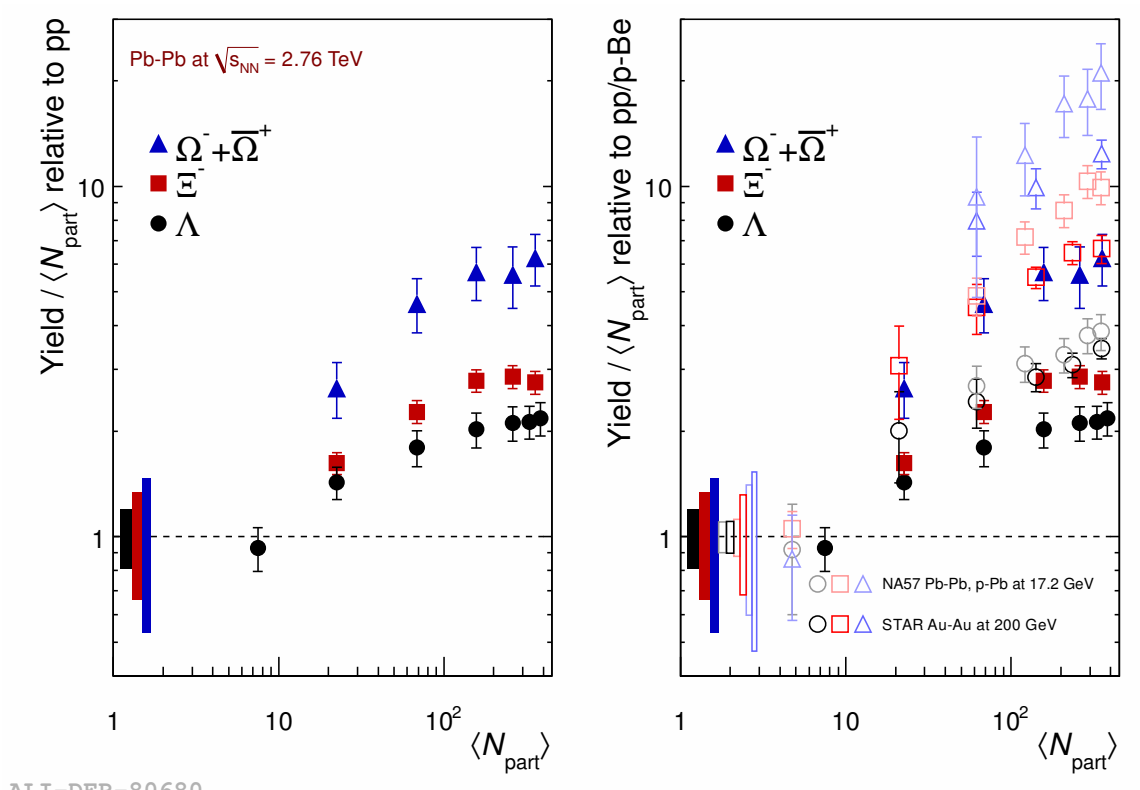

Figure 8. Left: strangeness enhancements for $\Lambda, \Xi^{-}$and $\Omega^{-}+\bar{\Omega}^{+}$as a function of the mean number of participants $\left\langle N_{\text {part }}\right\rangle$. Boxes on the dashed line at unity indicate statistical and systematic uncertainties on the pp reference. Right: comparison with lower energy measurements at SPS and RHIC (hollow symbols) [22].

Since the production rates of charged particles do not scale linearly with $\left\langle N_{\text {part }}\right\rangle$ [15], a way to factor out the enhancement due to strangeness content from the overall increase in charged-particle production is to compute the ratio of strange particles to pions. As reported in the next section, the $\Xi$ and $\Omega$ production rates have been compared with the $\pi$ yields in all the three collisions systems: the increase in the ratios of multi-strange particles to pion when going from central $\mathrm{Pb}-\mathrm{Pb}$ to $\mathrm{pp}$ is about half that of the standard enhancement ratio as defined above [22].

The nuclear modification factor $R_{\mathrm{AA}}$ is defined as the ratio between the $p_{\mathrm{T}}$ spectra in $\mathrm{Pb}-\mathrm{Pb}$ and those in pp scaled with the number of binary nucleon-nucleon collisions. In Fig. 9 the $R_{\mathrm{AA}}$ for pions, 
kaons and (anti-)protons are compared with those for multi-strange baryons, both in central (0-10\%) and peripheral $(60-80 \%) \mathrm{Pb}-\mathrm{Pb}$ collisions. For the most central events, the last two points at high $p_{\mathrm{T}}$ both for $\Xi$ and $\Omega$ have been obtained by extrapolating the corresponding reference pp spectra.
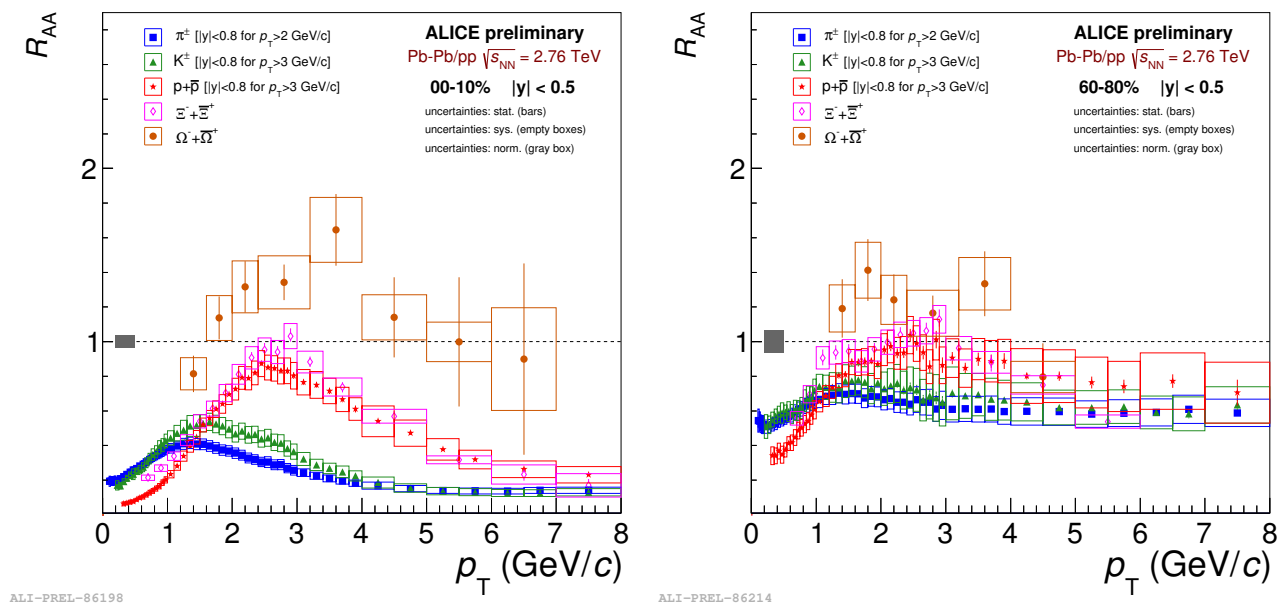

Figure 9. Nuclear modification factors $R_{\mathrm{AA}}$ for $\Xi$ and $\Omega$ compared to those for charged identified particles, for $0-10 \%$ (left) and $60-80 \%$ (right) centrality $\mathrm{Pb}-\mathrm{Pb}$ collisions at $\sqrt{s_{\mathrm{NN}}}=2.76 \mathrm{TeV}$.

The $R_{\mathrm{AA}}$ in the absence of nuclear or medium effects is by construction unity at high $p_{\mathrm{T}}$ where hard processes dominate. The particular shape differences of the $R_{\mathrm{AA}}$ for central collisions between baryons and mesons are a consequence of the interplay between the shapes of the particle $p_{\mathrm{T}}$ distributions, the collective flow (both depending on particle mass), jet-quenching phenomena (sensitive to parton type), and possible novel hadronization phenomena such as parton recombination. One easily identifies the similarities of the curves for two groups of particles (baryons and mesons), mass ordering at intermediate $p_{\mathrm{T}}$ and a mass-independent suppression above $8 \mathrm{GeV} / c$. The large $R_{\mathrm{AA}}$ values for the $\Omega$ can be related to the suppressed strangeness production in pp collisions. As expected, for peripheral events $R_{\mathrm{AA}}$ values closer to unity and reduced differences among the different species are observed.

\subsection{Results in $\mathrm{p}-\mathrm{Pb}$ collisions}

The transverse momentum spectra of $\pi, \mathrm{K}, \mathrm{p}$ and $\Lambda$ have been measured in different VZERO multiplicity classes in $\mathrm{p}-\mathrm{Pb}$ collisions at $\sqrt{s_{\mathrm{NN}}}=5.02 \mathrm{TeV}$ [33]. For $p_{\mathrm{T}}$ below $2 \mathrm{GeV} / c$ the spectra become harder as the multiplicity increases and the effect is stronger for heavier particles. This pattern is known from heavy-ion collisions where it is attributed to the hydrodynamical evolution of the medium and indeed also $p_{\mathrm{T}}$ spectra measured in high multiplicity $\mathrm{p}-\mathrm{Pb}$ collisions are better described by models which incorporate hydrodynamics. To further study the evolution of the spectral shapes with multiplicity a blast-wave analysis has been performed. The results are shown in Fig. 10: the two fit parameters, which in heavy-ion collisions are typically connected to the kinetic freeze-out temperature $T_{k i n}$ and the transverse velocity profile $\left\langle\beta_{\mathrm{T}}\right\rangle$, are compared for data from the three collision systems ( $\mathrm{pp}, \mathrm{Pb}-\mathrm{Pb}$ and $\mathrm{p}-\mathrm{Pb}$ ) and for pp events generated with PYTHIA 8. The behaviour in the data is qualitatively similar for all the systems: this may indicate the presence of radial flow or alternatively, as shown by the pp Monte Carlo generator, be due to other mechanisms such as color reconnection. 


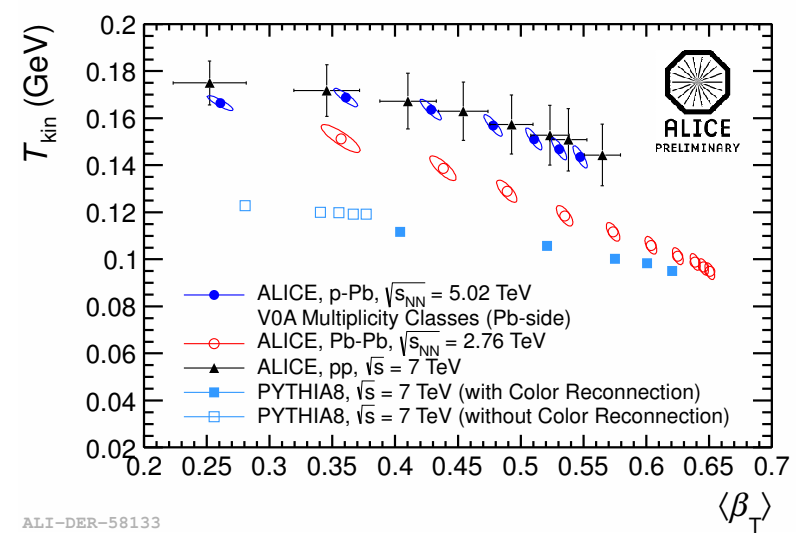

Figure 10. Results of blast-wave fits to the $p_{\mathrm{T}}$ spectra measured in $\mathrm{pp}, \mathrm{p}-\mathrm{Pb}$ and $\mathrm{Pb}-\mathrm{Pb}$ collisions and from PYTHIA 8 simulation of pp collisions (with and without color reconnection). Charged particle multiplicity increases from left to right.

As already mentioned for $\mathrm{Pb}-\mathrm{Pb}$, an appropriate way to factor out the strangeness enhancement from the overall increase in particle production is to compute the ratio of $\Xi$ and $\Omega$ production with respect to the $\pi$ yields. This has been done as a function of the average charged-particle multiplicity at mid-rapidity $\langle d \mathrm{~N} / d \mathrm{y}\rangle$ for all the three collisions systems, including $\mathrm{p}-\mathrm{Pb}$, as shown in Fig. 11 . The multi-strange baryon production relative to pions is shown to increase by up to a factor of about 3 when going from $\mathrm{pp}$ to $\mathrm{Pb}-\mathrm{Pb}$, reaching a saturation in the most central $\mathrm{Pb}-\mathrm{Pb}$ collisions at values predicted by statistical hadronization models for a chemical freeze-out temperature of about $155 \mathrm{MeV}$. In this picture, the $\mathrm{p}-\mathrm{Pb}$ results show monotonically increasing ratios, with $\Xi / \pi$ reaching the saturation level observed in central $\mathrm{Pb}-\mathrm{Pb}$ and $\Omega / \pi$ being not higher compared to the values for peripheral $\mathrm{Pb}-\mathrm{Pb}$ collisions. This indicates that thermal description of the observed multi-strange yields will thus be worse in $\mathrm{p}-\mathrm{Pb}$ than in $\mathrm{Pb}-\mathrm{Pb}$.
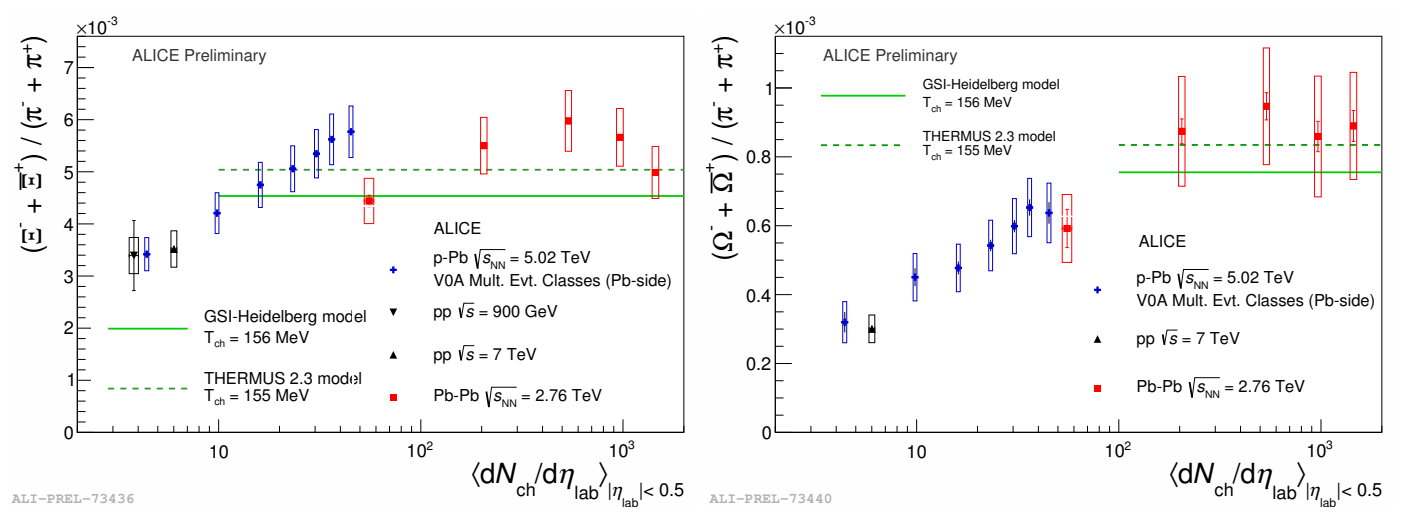

Figure 11. Multi-strange hyperon-to-pion ratios as a function of multiplicity (centrality) for $\mathrm{pp}, \mathrm{p}-\mathrm{Pb}$ and $\mathrm{Pb}-\mathrm{Pb}$ collisions shown together with predictions from statistical hadronization models. 
Measurements of the nuclear modification factors have been carried out also for $\mathrm{p}-\mathrm{Pb}$ collisions. Fig. 12 shows the $R_{\mathrm{pPb}}$ for charged $\pi, \mathrm{K}$ and p up to $20 \mathrm{GeV} / c$ and for $\Xi$ up to $6 \mathrm{GeV} / c$ in NSD p-Pb collisions. The nuclear modification factors for pions and kaons are flat over the full measured $p_{\mathrm{T}}$ range above $3 \mathrm{GeV} / c$ and do not differ from that of charged particles within the systematic uncertainties. At intermediate $p_{\mathrm{T}}$ (between 2 and $6 \mathrm{GeV} / c$ ) a mass ordering, with larger $R_{\mathrm{pPb}}$ for higher particle mass, is observed: in $\mathrm{Pb}-\mathrm{Pb}$ collisions this is interpreted as an effect due to radial flow. Only $\mathrm{p}$ and $\Xi$ exibit a Cronin peak, which indicates that the enhancement seen for charged particles is driven by protons. For larger transverse momenta $\left(p_{\mathrm{T}}>8 \mathrm{GeV} / c\right)$ all particle show the same $R_{\mathrm{pPb}}$ consistent with unity.

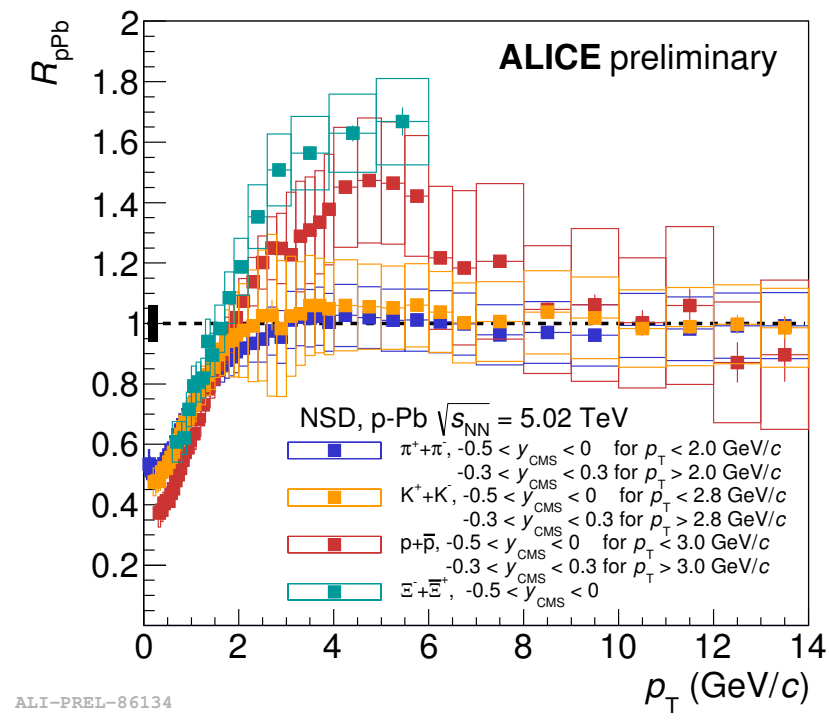

Figure 12. Nuclear modification factors $R_{\mathrm{pA}}$ for $\pi, \mathrm{K}, \mathrm{p}$ and $\Xi$ in NSD p-Pb collisions at $\sqrt{s_{\mathrm{NN}}}=5.02 \mathrm{TeV}$.

\section{Conclusions}

The ALICE experiment has performed a systematic study of the strange and light identified charged particles in a wide range of $p_{\mathrm{T}}$ for events collected from the three collision systems during the first $\mathrm{LHC}$ run phase. Measurements in pp not only provide the baseline for understanding results in $\mathrm{Pb}-\mathrm{Pb}$ and $\mathrm{p}-\mathrm{Pb}$ collisions: transverse momentum spectra for strange and multi-strange, for instance, play a key role in setting constrains on the Monte Carlo generator models.

In central $\mathrm{Pb}-\mathrm{Pb}$ collisions $\pi, \mathrm{K}$ and $\mathrm{p}$ production is reduced at high $p_{\mathrm{T}}$ when compared with expectations from the corresponding pp reference spectra, an effect which can be interpreted in terms of jet quenching. Both strange and identified particle spectra are qualitatively well reproduced by hydrodynamical models, while the $p_{\mathrm{T}}$-integrated yields fit the predictions from statistical hadronization with a chemical freeze-out temperature around $155 \mathrm{MeV}$. The strangeness enhancement, one of the many observables traditionally related to the QGP phase, has been observed also at the LHC, though reduced compared to that at lower energies due to easing of canonical suppression in pp collisions. Nuclear modification factors show mass ordering at intermediate $p_{\mathrm{T}}$, while no mass dependence is 
seen at higher momenta indicating that the chemical composition of leading particles from jets in the medium is similar to that of vacuum jets. Measurements in $\mathrm{p}-\mathrm{Pb}$, initially conceived as a control experiment only, have shown $\pi, \mathrm{K}$ and $\mathrm{p}$ spectra with a shape evolution with multiplicity with similar flow signatures as in $\mathrm{Pb}-\mathrm{Pb}$ : hydrodynamic calculations give the best decription of the highmultiplicity $p_{\mathrm{T}}$ spectra also in $\mathrm{p}-\mathrm{Pb}$ collisions, although the same evolution could also be explained by other mechanisms such as color reconnection that mimics radial flow effects in pp.

\section{References}

[1] B. Müller, J. Schukraft and B. Wyslouch, Ann. Rev. Nucl. Part. Sci. 62, 361 (2012).

[2] R. J. Fries, V. Greco and P. Sorensen, Ann. Rev. Nucl. Part. Sci. 58, 177 (2008).

[3] S. Sapeta and U.A. Wiedemann, Eur. Phys. J. C 55, 293 (2008).

[4] J. Rafelski and B. Müller, Phys. Rev. Lett. 48, 1066 (1982).

[5] P. Koch, B. Müller and J. Rafelski, Phys. Rep. 142, 167 (1986).

[6] K. Aamodt et al., JINST 3, S08002 (2008).

[7] B. Abelev et al., Int. J. Mod. Phys. A 291430044 (2014).

[8] K. Aamodt et al., Eur. Phys. J. C 71, 1594 (2011).

[9] B. Abelev et al., Phys. Lett. B 712, 309 (2012).

[10] G. Volpe for the ALICE Collaboration, these proceedings.

[11] P. Z. Skands, Phys. Rev. D 82, 074018 (2010).

[12] R. Skands, arXiv:1010.3558v1, (2010).

[13] D. Colella for the ALICE Collaboration, J. Phys. Conf. Ser. 509, 012090 (2014).

[14] B. I. Abelev et al., Phys. Rev. C 75, 064901 (2007).

[15] K. Aamodt et al., Phys. Rev. Lett. 106, 032301 (2011).

[16] B. Abelev et al., Phys. Lett. B 736, 196 (2014).

[17] B. Abelev et al., Phys. Rev. C 88, 044910 (2013).

[18] C. Shen, U. Heinz and P. Huovinen and H. Song, Phys. Rev. C 84, 044903 (2011).

[19] Y. Karpenko and Y. Senyukov, J. Phys. G 38, 124059 (2011).

[20] P. Bozek, Phys. Rev. C 85, 034901 (2012).

[21] K. Werner, Phys. Rev. C 85, 064907 (2012).

[22] B. Abelev et al., Phys. Lett. B 728, 216 (2014).

[23] J. Cleymans, I. Kraus, H. Oeschler, K. Redlich and S. Weaton, Phys. Rev. C 74, 034903 (2006).

[24] A. Andronic et al., Nucl. Phys. A 772, 167 (2006).

[25] M. Petran, J. Letessier, J. Rafelski and G. Torrieri, Comput. Phys. Commun. 185, 2056 (2014).

[26] F. Becattini et al., Phys. Rev. Lett. 111, 082302 (2013).

[27] M. Petran, J. Letessier, V. Petracek and J. Rafelski, Phys. Rev. C 88, 034907 (2013).

[28] C. Ratti, R. Bellewied, M. Cristoforetti and M. Barbaro, Phys. Rev. D 85, 014004 (2012).

[29] A. Andronic et al., Nucl. Phys. A 904-905, 535c (2013).

[30] F. Antinori et al., J. Phys. G 32, 427 (2006).

[31] F. Antinori et al., J. Phys. G 37, 045105 (2010).

[32] B. I. Abelev et al., Phys. Rev. C 77, 044908 (2008).

[33] B. Abelev et al., Phys. Lett. B 728, 25 (2014). 